\title{
3D cardiac Chemical Shift Imaging of [1-13C] hyperpolarized acetate and pyruvate in pigs
}

\author{
Luca Menichetti ${ }^{1,2}$, Francesca Frijia ${ }^{2 *}$, Alessandra Flori ${ }^{3}$, Vincenzo Lionetti ${ }^{3}$, Matteo Liserani ${ }^{4}$, Giulio Giovannetti ${ }^{1}$, \\ Giacomo Bianchi ${ }^{2}$, Simone L Romano ${ }^{3}$, Vincenzo Positano ${ }^{2,1}$, Jan Henrik Ardenkjaer-Larsen ${ }^{5,6}$, Rolf F Schulte ${ }^{7}$, \\ Fabio A Recchia ${ }^{2}$, Luigi Landini ${ }^{8}$, Maria Filomena Santarelli, ${ }^{1,2}$, Massimo Lombardi ${ }^{2}$
}

From 16th Annual SCMR Scientific Sessions

San Francisco, CA, USA. 31 January - 3 February 2013

\section{Background}

13C Dynamic Nuclear Polarization (DNP) with rapid dissolution together with Magnetic Resonance Chemical Shift Imaging (CSI) have been used for non-invasive real-time metabolic assessment in cardiac experimental models on a clinical 3T scanner. Here, we report an in vivo comparison of hyperpolarized [1-13C] pyruvate and [1-13C] acetate perfusion and metabolism: a method based on a 3D Spiral CSI sequence is presented for obtaining spatially and spectrally-resolved information on whole heart cardiac metabolism.

\section{Methods}

In this work hyperpolarized [1-13C] pyruvate and [1-13C] acetate were injected in vivo to obtain spatially and spectrally resolved information of basal metabolism on whole heart in middle size animal models. Five healthy male farm pigs $(38 \pm 2 \mathrm{~kg})$ were studied in basal condition and subjected to imaging experiments performed on a $3 \mathrm{~T} \mathrm{GE}$ Signa HDx scanner using a 13C-quadrature birdcage coil (Rapid Biomedical). An HyperSense DNP polarizer (Oxford Inst.) was employed for the studies: a procedure for the hyperpolarization and dissolution of a large dose of TRIS-[1-13C]acetate water/glycerol mixture was set up while the preparation of a large dose of [1-13C] pyruvic acid was performed as recently published by this group. An anatomical region of interest covering the whole heart was first acquired with a proton reference scan and the metabolic information was then obtained using 3D IDEAL spiral CSI on the same region. Image re-slicing along cardiac short axis (SA) views and image fusion of $13 \mathrm{C}$ metabolite maps and anatomical $1 \mathrm{H}$ reference images were performed by PMOD software.

\section{Results}

A graph of the $\gamma$-variate and mono-exponential fitting of hyperpolarized [1-13C] acetate myocardial spectroscopic signals is reported in Figure 1 while a representative map in SA orientation through the heart is shown in Figure 2: [1-13C] acetate is extracted inside the heart and clearly detected in the heart-chambers and myocardial wall. Representative maps of spatial distribution of [1-13C] bicarbonate, [1-13C] lactate and [1-13C] pyruvate in SA orientation through the heart are also produced using hyperpolarized [1-13C] pyruvate.

\section{Conclusions}

A comparison between acetate and pyruvate 13C-mapping has been realised as far as we know for the first time in pigs with this experimental approach. This ongoing study demonstrates the feasibility of whole-heart $13 \mathrm{C}$-cardiac metabolic imaging in pigs for detecting and mapping cardiac metabolism in basal condition with hyperpolarized [1-13C]acetate in comparison with [1-13C] pyruvate.

This study is the first step towards the optimization of the $[1-13 C]$ acetate concentration and the acquisition sequence parameters to ensure suitable MR signals in myocardial tissue and to study its metabolic fate.

\section{Funding}

Self funding. 


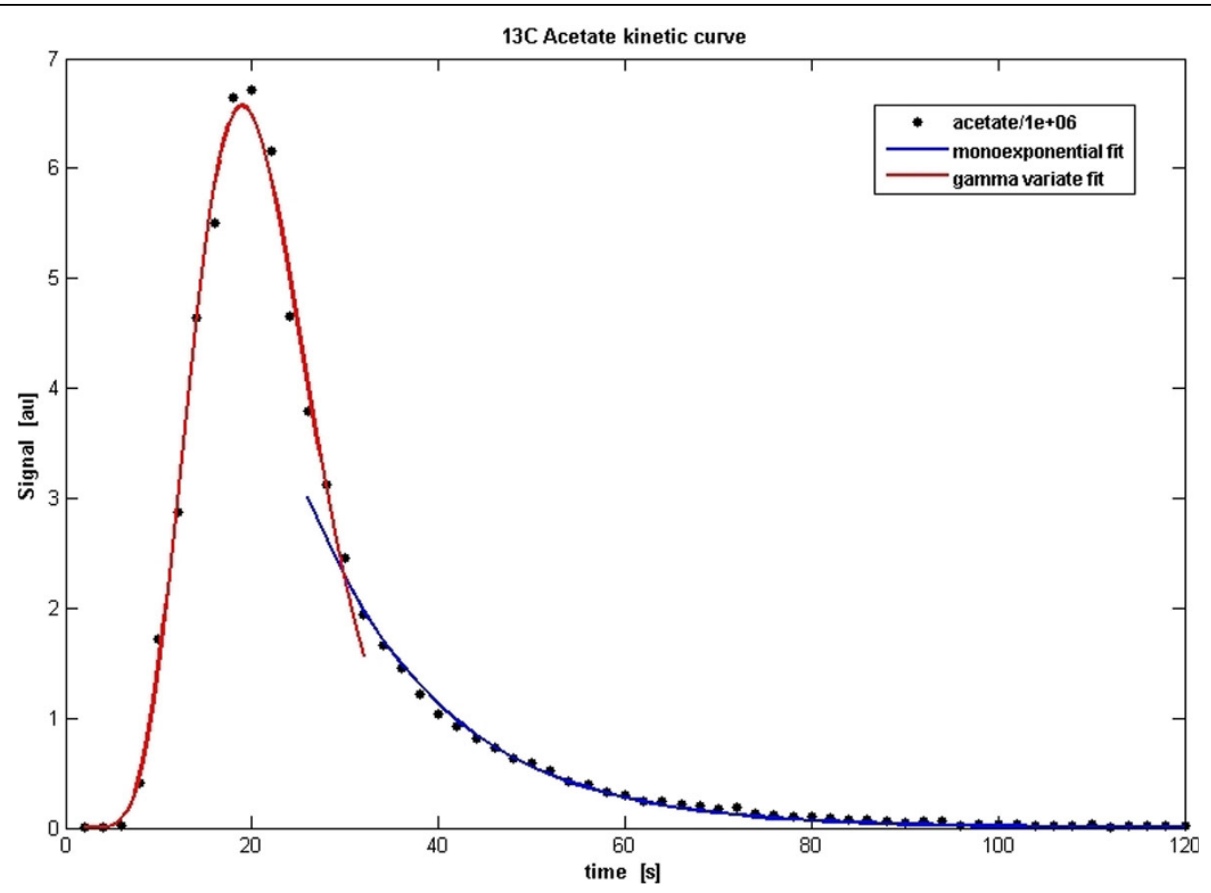

Figure 1 13C dynamic spectra were acquired using a slice selective pulse-and-acquire sequence (bandwidth $5000 \mathrm{~Hz}, 2048$ pts, $10^{\circ} \mathrm{FA}$ ). A longaxis slice of $20 \mathrm{~mm}$ was selected during excitation. Spectra were acquired from the beginning of the injection of the hyperpolarized [1-13C] acetate, every $2 \mathrm{~s}$, for $120 \mathrm{~s}$. Diagrammatic representation of the $\gamma$-variate and mono-exponential fitting of cardiac spectroscopic signal to obtain rate constants $(\mathrm{N}=4)$.

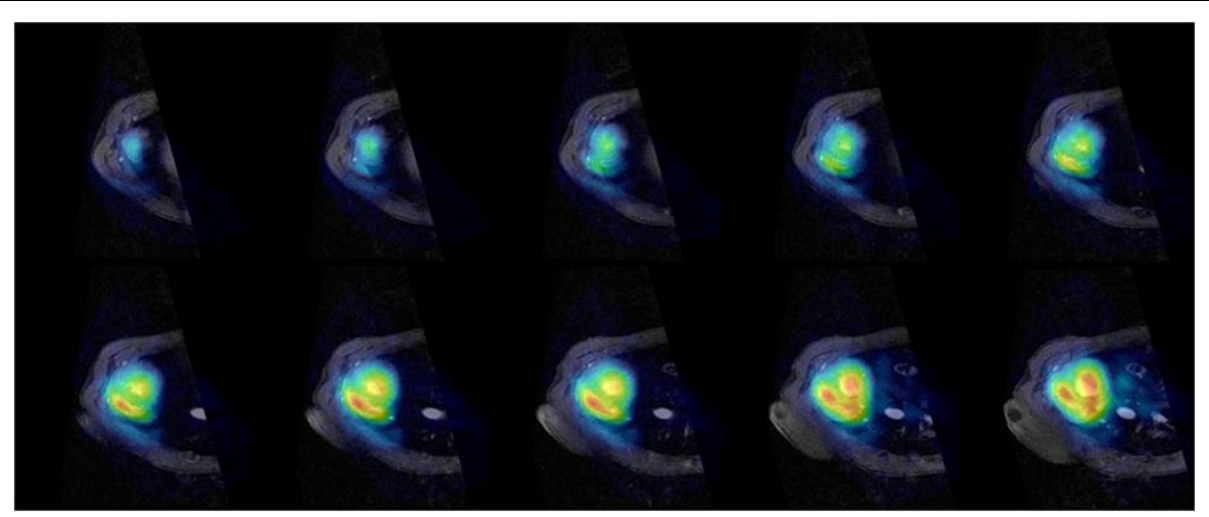

Figure 2 Representative maps in SA view of the heart showing the in vivo spatial distribution of hyperpolarized TRIS-[1-13C] acetate in pigs; spectroscopic data were normalized to the maximum value of signal amplitude.

\section{Author details}

'Institute of Clinical Physiology, National Research Council, Pisa, Italy. ${ }^{2}$ Fondazione G. Monasterio CNR-Regione Toscana, Pisa, Italy. ${ }^{3}$ Scuola Superiore Sant'Anna, Pisa, Italy. ${ }^{4}$ Faculty of Physics, University of Pisa, Pisa, Italy. ${ }^{5} \mathrm{GE}$ Healthcare, Hillerod, Denmark. ${ }^{6}$ Department of Electrical Engineering, Technical University of Denmark, Kongens Lyngby, Denmark. ${ }^{7} \mathrm{GE}$ Global Research, Munich, Germany. ${ }^{8}$ Department of Information Engineering, University of Pisa, Pisa, Italy.

Published: 30 January 2013
doi:10.1186/1532-429X-15-S1-P10

Cite this article as: Menichetti et al:: 3D cardiac Chemical Shift Imaging of [1-13C] hyperpolarized acetate and pyruvate in pigs. Journal of Cardiovascular Magnetic Resonance 2013 15(Suppl 1):P10. 